\title{
Formation of prismatic dislocation loops during unloading in nanoindentation
}

\author{
Haiyang Yu ${ }^{\mathrm{a}, *}$, Alan Cocks ${ }^{\mathrm{b}}$, Edmund Tarleton ${ }^{\mathrm{b}, \mathrm{a}}$ \\ ${ }^{a}$ Department of Materials, University of Oxford, Parks Road, Oxford OX1 3PH, UK \\ ${ }^{b}$ Department of Engineering Science, University of Oxford, Parks Road, Oxford OX1 3PJ, UK
}

\begin{abstract}
The formation of prismatic dislocation loops during nanoindentation of bcc iron is simulated with discrete dislocation plasticity. Interestingly, it is not the loading phase, but the unloading phase of the test which is found to be crucial for prismatic loop formation when indenting a (001) crystal surface. In this case, prismatic loops do not form during loading, but develop as the indenter is removed, in contrast to (111) indentation where prismatic loops are nucleated almost immediately upon loading.
\end{abstract}

Keywords: nanoindentation, prismatic loops, discrete dislocation dynamics

Prismatic (dislocation) loops are commonly observed during nanoindentation, which is a popular method for probing the mechanical properties of small volumes of material. During indentation, a confined zone of high dislocation density develops under the indenter, from which prismatic loops and/or helical dislocations can be emitted into the bulk, forming several 'arms' along the active slip directions [1]. These arms are long relative to the size of the high density zone. The formation of prismatic loops has been observed in a variety of crystal structures, such as $\mathrm{Cu}[2,3]$, Ta [4] and $\alpha-\mathrm{Ti}$ [5]. The arrangement of prismatic loops and the role they play in plastic deformation depend on the crystal structure and indentation direction. In bcc Fe single crystals [6], there are four inclined $\langle 111\rangle$ directions when indenting on the (100) surface, along which interstitial prismatic loops move downwards into the bulk. With corresponding vacancy loops exiting at the free surface, an indent impression surrounded by a surface pileup with four-fold symmetry is left on the indentation surface $[7,8]$. Indentation into a (110) surface generates two traces along the $\langle 111\rangle$ directions, which lie entirely in the (110) plane. Half-loops terminating at the free surface transport material away from the tip without producing any pileup, forming a dislocation rosette visible on the surface. The other two $\langle 111\rangle$ directions are inclined into the bulk and both intersect the (110) plane along $\langle 100\rangle$, which can lead to a visible surface pileup along this direction. These conclusions are consistent with the experimental observations on bcc Ta [7] and W [8]. Similar phenomena are also observed in $\mathrm{Cu}$ [2], where a long extended rosette of prismatic loops is observed on the surface under (111) indentation. Prismatic loops are usually emitted after a zone with high dislocation density has developed beneath the indenter, acting to accommodate the applied deformation and extend the plastic zone. When indenting a (111) surface of a bcc crystal, the emis-

\footnotetext{
${ }^{*}$ Corresponding author

Email addresses: haiyang. yun@materials.ox.ac.uk (Haiyang Yu ), alan.cocks@eng.ox.ac.uk (Alan Cocks), edmund.tarleton@eng.ox.ac.uk (Edmund Tarleton)
}

sion of complete prismatic loops can occur at a much lower dislocation density, which is then associated with a pop-in on the force-displacement curve.

The formation mechanism of prismatic loops has been studied mainly by molecular dynamics (MD) simulations. Alcalá et al. [9] found that twin nucleation and interaction as well as annihilation produce prismatic loops, using Ta as a model material. Roy et al. [10] highlighted the importance of collinear reactions, which produce v-shaped dislocations which then interact and form prismatic loops. This dislocation mechanism was later confirmed experimentally by Lee et al. [11] in Au. Another prismatic loop formation mechanism involves the crossslip and self-annihilation of a single shear loop. As demonstrated by Remington et al. [4], also using Ta, the screw components of a shear loop cross-slip several times generating a quasi-cylindrical surface, the opposite segments then approach and eventually pinch off into a prismatic loop. This " lasso"like mechanism operates widely; including in $\mathrm{Au}$ [11], Ti and Mg [12], AlN (with the wurtzite structure) [13] and VN [14]. While providing important atomistic insights, MD simulations are limited in length and time scales. The size of the simulation box in nanoindentation simulations of prismatic loop formation as reviewed above is below $100 \mathrm{~nm}^{3}$ (although MD simulations of nanoindentation can be larger, but typically less than $1 \mu \mathrm{m}^{3}$ ), and the size of prismatic loops is around $10 \mathrm{~nm}$, which is smaller than those observed experimentally, whose radii can be larger than $100 \mathrm{~nm}[15,1]$. Discrete dislocation dynamics (DDD) $[16,17]$ has proven effective in simulating the formation of prismatic loops at length and time scales not currently accessible by atomistic simulations. Gagel et al. [1] simulated the formation of prismatic loops in $\mathrm{Al}$, achieving good agreement with experimental observations [2]. Roy et al. [10] simulated nanoindentation of Au particles both with DDD and MD. They employed MD to investigate the prismatic loop formation mechanism and DDD to reach the experimental dimensions. So far, DDD has only been used to simulate prismatic loop formation during nanoindentation in fcc material $[1,10]$. In the bcc 


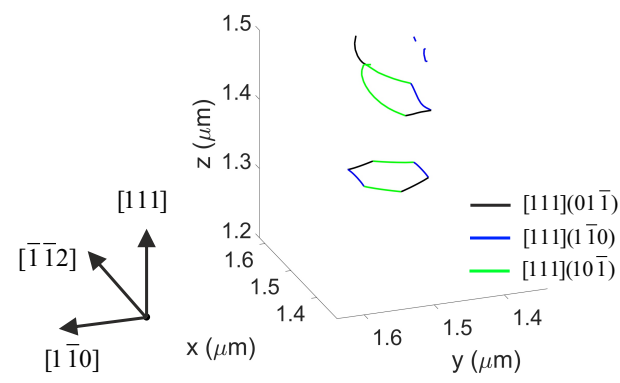

(a)

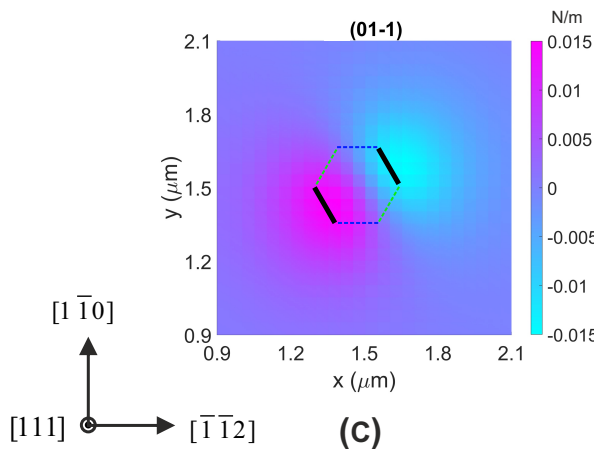

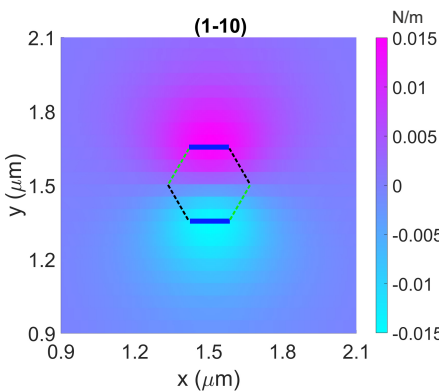

(b)

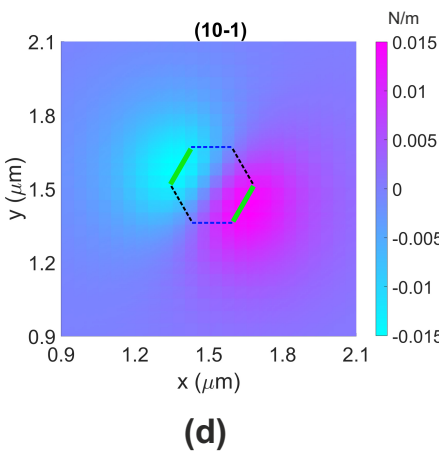

Figure 1: (a) The early stage of (111) indentation showing a complete prismatic loop and the formation of a second, at an indentation depth of 2.2 nm. (b-d) Peach-

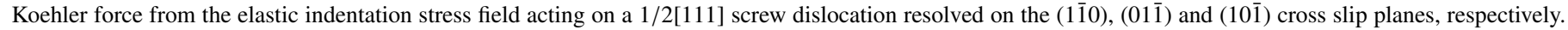
The contours are projected on the (111) plane, $0.3 \mu \mathrm{m}$ below indentation surface. An illustration of the projection plane is found in Figure S1(a) in the Supplementary data. The solid lines illustrate the favoured cross-slip planes favoured in the stress regions, following the colour code in figure (a), and the dashed lines indicate the rest of the hexagonal prismatic loop.

case, it has been employed to simulate prismatic loop generation from defects [18], where a very similar "lasso"-like mechanism assisted by cross-slip applies.

The nature of prismatic loop formation is dependent on the loading conditions, with loading direction, tip size, indentation depth and loading rate being examined extensively $[4,1,10]$. Surprisingly, little attention has been paid to unloading. Recent work by Lee et al. [11] observed prismatic loop formation in situ during nanoindentation of Au nanowires. However, dislocation structures generated during indentation are usually examined ex situ, where unloading effects are essential. In this work, we simulate prismatic loop formation during spherical nanoindentation of Fe with DDD to investigate the unloading stage.

The radius of the indenter is $R=1 \mu \mathrm{m}$, and the dimensions of the sample are $3 \times 3 \times 1.5 \mu^{3}$. Initially, a Frank-Read (FR) source is placed near the indentation surface, the source is a square dislocation loop with one edge free and the other edges fixed. Finite boundary conditions are implemented using the superposition principle [19]. Details of the framework are in [20]. Indentation is idealised as Hertzian contact between a sphere and a semi-infinite elastic medium, which is a reasonable approximation for small deformation [15]. For a desired indentation depth, we evaluate the contact radius and Hertzian pressure, the pressure is then distributed over the finite element nodes in the contact region using the element shape function [1]. Dislocations gliding on the $\{110\}$ planes are considered and cross slipping between these planes is allowed. Following Mun- day et al. [18], cross-slip of a screw dislocation occurs when the resolved shear stress on one of the $\{110\}$ planes exceeds that on the current glide plane, which maximizes the energy dissipation assuming the same dislocation mobility on all the planes. Material parameters relevant to bcc Fe are used: lattice constant $a=2.856 \AA$, shear modulus $G=76 \mathrm{GPa}$, Poisson's ratio $v=0.3$.

Two crystal orientations were simulated; indentation into the (001) surface and the (111) surface. In the (001) case, the $x, y$ and $z$ axes in the global coordinate system were aligned with the crystal directions [100], [010] and [001]; in the (111) case, the $x, y$ and $z$ axes were aligned with the crystal directions [1 $1 \overline{1} 2],[1 \overline{1} 0]$ and [111], respectively. The top surface was indented along the negative $z$ direction. Of most interest are slip systems with a component of the slip direction along $z$, as these can accommodate the applied load. These include all four $\langle 111\rangle$ slip directions in (001) indentation and a dominant $\langle 111\rangle$ slip direction perpendicular to the surface in (111) indentation. The other three slip directions in (111) indentation form a shallow angle $\left(19.5^{\circ}\right)$ with the free surface.

In the (111) indentation simulation, an FR source with a length of $l_{0}=49.5 \mathrm{~nm}$ and Burgers vector $\boldsymbol{b}=1 / 2$ [111] was placed $30 \mathrm{~nm}$ below the surface, offset by $74 \mathrm{~nm}$ from the indentation axis. An array of prismatic loops was formed on a hexagonal glide prism along the indentation axis by the intersection of the three (110) cross slip planes with the (111) plane, as shown in Figure 1(a) and Movie 1 in Supplementary data. The prismatic loops were generated from a shear loop via multi- 

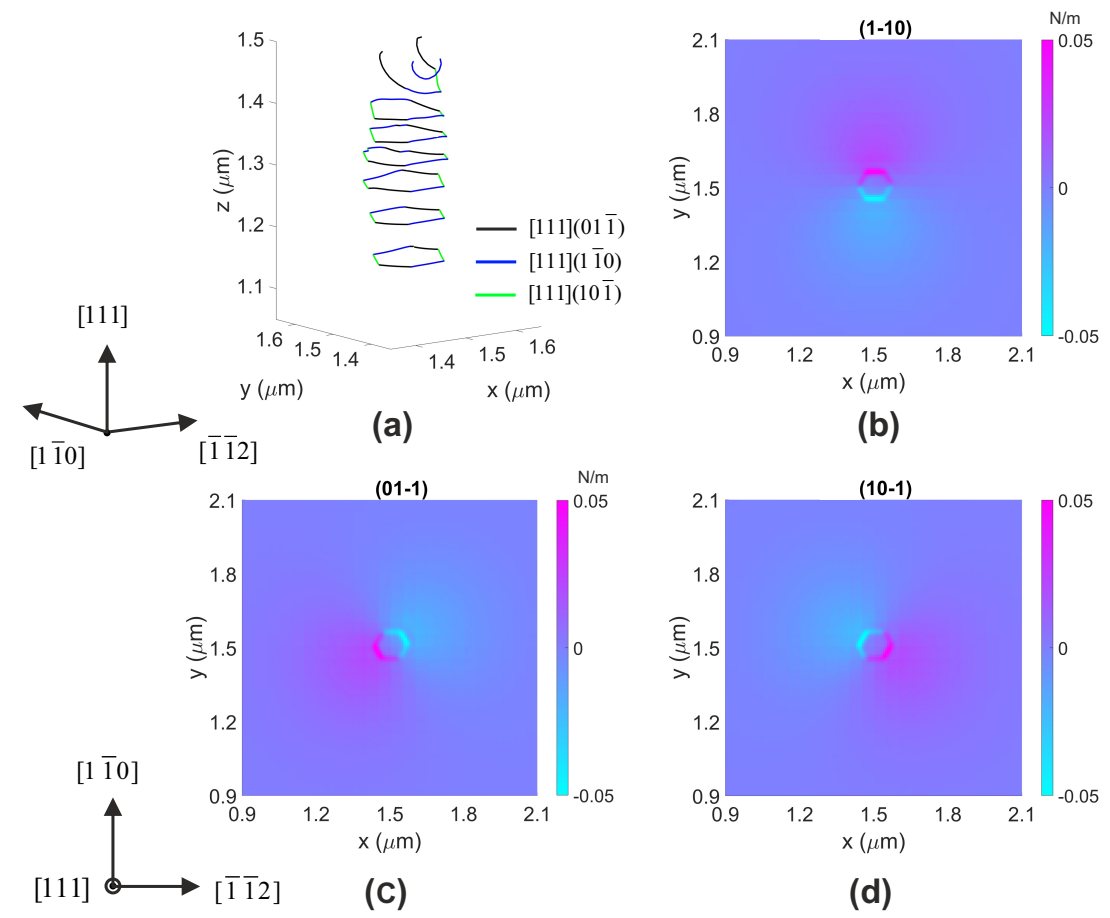

Figure 2: (a) (111) indentation at an indentation depth of $3.4 \mathrm{~nm}$, and six interstitial prismatic loops have formed. (b-d) Peach-Koehler force from the total stress

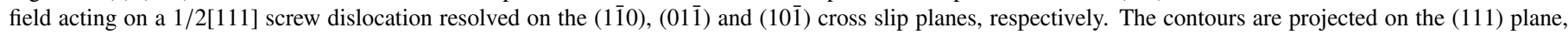
$0.3 \mu \mathrm{m}$ below indentation surface. An illustration of the projection plane is found in Figure S1(a) in the Supplementary data.

ple cross-slip and pinch-off events, the mechanism is very similar to that observed by Gagel et al. [1] in their simulations. The stress field underneath the indenter, as shown in Figure 1, is characterised in terms of the Peach-Keohler force acting on a [111] screw segment resolved on the three relevant $\{1 \overline{1} 0\}$ cross slip planes, $\boldsymbol{F} \cdot \boldsymbol{m}$, where $\boldsymbol{F}=(\boldsymbol{\sigma} \cdot \boldsymbol{b}) \times \boldsymbol{l}$ is the Peach-Keohler force and $\boldsymbol{m}=\boldsymbol{l} \times \boldsymbol{n}$, with $\boldsymbol{l}$ being the unit line direction and $\boldsymbol{n}$ the cross slip plane normal. $\sigma$ is evaluated on a finite element mesh discretizing the sample into $60 \times 60 \times 30$ elements. Contours of the resolved cross slip forces are evaluated on the (111) habit plane of the forming prismatic loop and display a threefold symmetry. The two lobes in Figure 1(b) show the regions where cross-slip on the (11) plane is favourable, Figure 1(c)

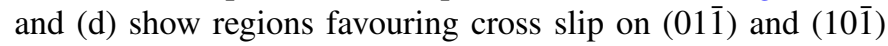
planes.

Upon loading, the FR source on the (110) plane first emits a shear loop; as the loop expands, the screw segments enter the purple regions in Figure 1(c) and (d), causing them to cross slip on the $(01 \overline{1})$ and $(10 \overline{1})$ planes, respectively; a second cross-slip event happens when the screw segments enter the cyan regions in Figure 1(c) and (d), followed by a third cross-slip event in the purple region in Figure 1(b), bringing the segments back onto the (110) plane. The opposite screw segments then approach and pinch off leaving a complete hexagonal interstitial prismatic loop which is repelled into the bulk of the material by the indentation pressure field. The other parts of the shear loop exit at the free surface during the expansion and multiple cross-slip events, leaving hexagonal surface steps, as if a vacancy prismatic loop of the same size had exited. The stress field of the interstitial loop does not break the three-fold symmetry of the total stress field. As shown in Figure 2, perfect symmetry is maintained after six prismatic loops are generated, as the stress field exerted by these loops has a hexagonal pattern. Therefore, this prismatic loop nucleation mechanism can repeat continuously. In the simulation shown in Movie 1 in Supplementary data, seven prismatic loops were generated. The process resembles the mechanism revealed by Gagel et al. [1] in (110) indentation in fcc materials where the prismatic loops were rhombus shaped, as there are two relevant slip planes and the stress field has a two-fold symmetry, in contrast to the hexagonal prismatic loops in the bcc case. Hexagonal prismatic loops were also observed by Munday et al. [18] in a bcc material due to the stress field generated by a spherical defect.

Considering that all the four $\langle 111\rangle$ slip directions in the case of (001) indentation are equivalent, we selected an FR source

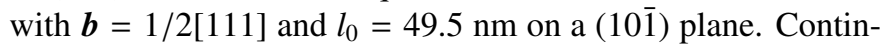
uous generation of prismatic loops as seen in (111) indentation did not occur for this loading condition. Among the various cases tested, generation of a complete prismatic loop was a rare event at the shallow indentation depths simulated here. In most cases, helical dislocations were observed, with only the occasional generation of a complete prismatic loop after a complex dislocation structure has formed. The resolved cross slip forces beneath the surface are shown in Figure 3. Sub-figures (a-c) illustrate the dislocation free forces the first dislocation loop is subjected to. The stress fields are not symmetric, with the upper region much less pronounced. Dislocation motion is mainly limited to the $(10 \overline{1})$ and $(01 \overline{1})$ planes; cross-slip onto the (1 $\overline{1} 0)$ 


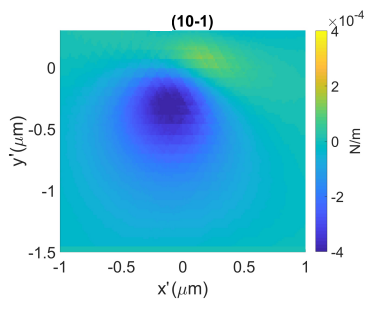

(a)

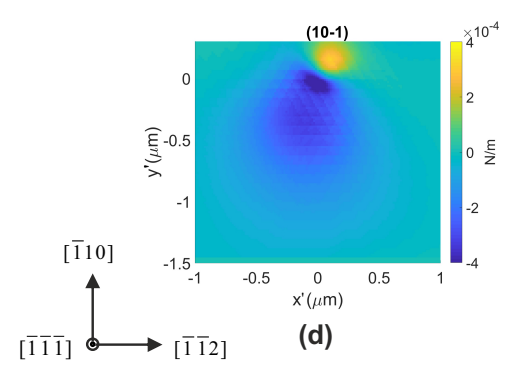

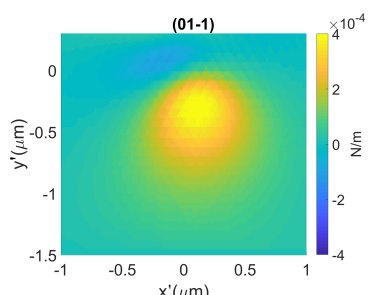

(b)

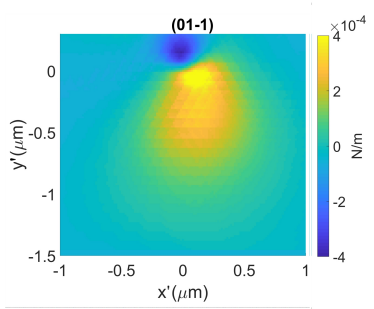

(e)

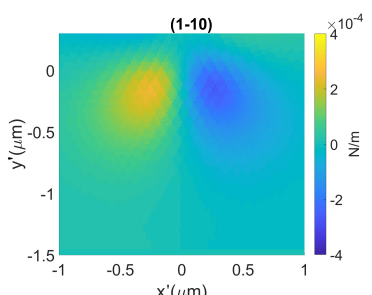

(c)

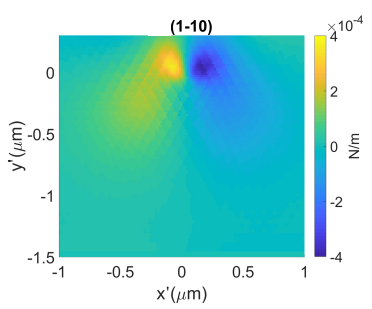

(f)

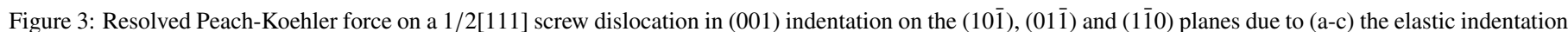
(Hertzian) stress field and (d-f) the total stress field including the dislocation stress field. The corresponding indentation depth is $4.3 \mathrm{~nm}$, eight half loops have been generated, with a pattern similar to that in Figure 4(a) but no helical dislocation has been formed. The contours are plotted in a projection on a plane normal to the Burgers vector, the distance from the plane to the centre of the indentation surface along the Burgers vector direction is $0.3 \mu m$. An illustration of the projection plane is found in Figure S1(b) in the Supplementary data.

plane could happen in some cases, but no further cross-slip and pinch-off was observed, which results in the generation of a

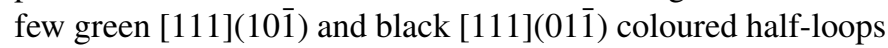
as shown in Figure 4(a), but with few blue [111](1110) coloured dislocations and no helical dislocations at the beginning of indentation. This is in contrast to (111) indentation where a complete prismatic loop could be emitted from the first shear loop. As more dislocations were generated, the form of the total stress field changed, as shown in Figure 3(d-f). The stress in the upper region, near the free surface, increases allowing the screw components to cross slip back onto the initial glide planes, as illustrated in Figure 4(a). However, the formation of a complete prismatic loop is rare, since the stress field keeps changing with further dislocation generation, adding to the complexity and uncertainty of dislocation interactions.

The structure in Figure 4(a) was then subjected to unloading. In Figure 4(b), dislocations have glided back towards the indenter due to the change in the local Peach-Koehler forces arising from the reduction of the contact pressure. During unloading, the long dislocation lines marked in Figure 4(a) have retracted and met the helices, as shown in Figure 4(b). These then pinch off to form pairs of interstitial prismatic loops, as shown in Figure 4(b-d). The mechanism can be seen more easily in Movie 2 in Supplementary data.

Prismatic loop formation during unloading rather than loading, was consistently observed in the cases tested, so it should be a feature of shallow (001) indentation in bcc. The prismatic loops (observed after indentation) may therefore not be related to the onset of plastic deformation, especially the initial pop-in in the (001) indentation case, as these are formed during the unloading stage. The rare formation of a prismatic loop during loading could only happen after sufficient dislocations have been generated, far beyond the initial pop-in. In contrast, the prismatic loops can be related to the initial pop-in in (111) indentation, since these can be continuously generated from the early stage indentation. In (001) indentation, plastic slip steps were left on the free surface by the parts of the shear loops that had exited during indentation. During unloading (in the absence of dislocation junction and tangle formation), the rest of the shear loops, including the helical dislocations that had moved into the bulk, retracted and exited from the surface, cancelling out some of the previously formed slip steps (plastic recovery). The prismatic loops formed during unloading kept moving into the bulk instead of flowing back (Movie 2 in Supplementary data), due to the repulsive forces between the interstitial prismatic loops which have moved far away into the bulk. A similar phenomenon was observed in [1]. This prevents some surface slip steps from being cancelled out, therefore, an indent imprint on the surface is observed after unloading. An area of positive surface deformation surrounding the imprint is also observed, but the magnitude of deformation is much smaller compared to the experiment where the indentation depth can be 10 times larger than that applied in the current simulation. It should be clarified that the surface imprint after unloading discussed here is not due to the prismatic loops visible in Figure 4(d), but due to their counterparts that have left the volume, i.e. it is the history of dislocation motion rather than the current configuration that determines the surface deformation [21]. It should also be noted that the current simulation adopts an idealised model considering just one slip system to highlight the prismatic loop formation process. In reality, dislocation junction and entanglement can also prevent dislocations from leaving the volume during unloading. This mainly happens in the vicinity of the indent, so it does not affect the prismatic loop formation mech- 


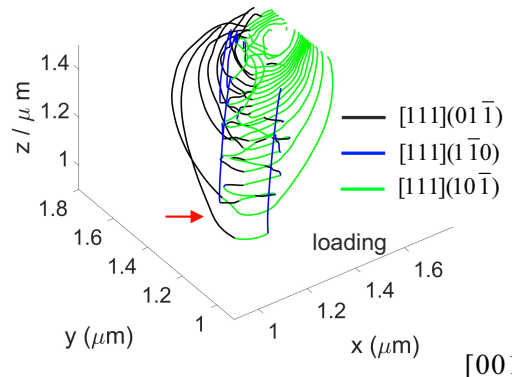

(a)

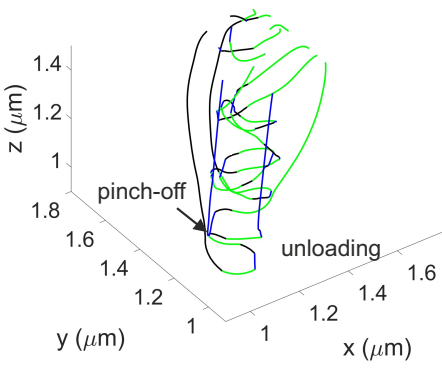

(b)

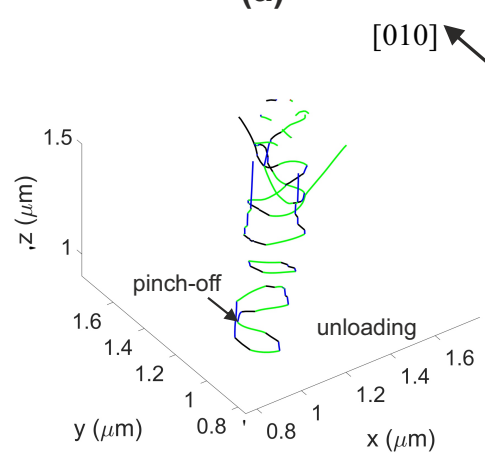

(c)
[100]

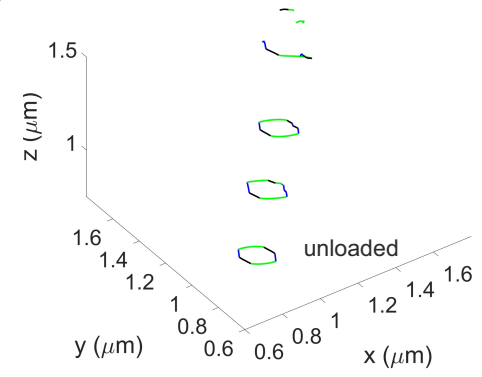

(d)

Figure 4: (a) The dislocation structure just before unloading, at an indentation depth of $7.3 \mathrm{~nm}$ along [001]; the red arrow highlights the first half loop which retracted and caused pinch-off during unloading. (b) The dislocation structure during unloading, where the first pinch-off occurred as the result of a retracting half loop intersecting with the helical dislocation. (c) The final pinch-off which led to the formation of three prismatic loops. (d) The prismatic loops left in the bulk after a long time of relaxation. The x,y,z directions are aligned with the crystal directions [100], [010] and [001], respectively, indentation is performed along the negative $\mathrm{z}$ direction.

anism, which can occur far away from the indent.

In summary, the formation of prismatic loops during nanoindentation of bcc material was simulated with DDD. The continuous emission of hexagonal prismatic loops during loading in (111) indentation was observed, which was attributed to the symmetry of the stress field. In contrast, prismatic loop formation in (001) indentation occurred only during unloading, due to complex dislocation kinematics. Prismatic loops in (001) indentation therefore have little to do with the initial pop-in as commonly postulated, but contribute partly to maintaining surface deformation after unloading. This work highlights the importance of considering the unloading phase in nanoindentation simulations.

\section{Acknowledgements}

This work was supported by the Engineering and Physical Sciences Research Council under Programme grant EP/L014742/1 and Fellowship grant EP/N007239/1.

\section{References}

[1] J. Gagel, D. Weygand, P. Gumbsch, Acta Mater. 111 (2016) 399-406.

[2] Y. Miura, J. Appl. Phys. 43 (1972) 2917-2918.

[3] Y. Miura, H. Nakamura, H. Kaieda, Trans. Jpn. Inst. Met. 17 (1976) 793798.
[4] T. P. Remington, C. J. Ruestes, E. M. Bringa, B. A. Remington, C. H. Lu, B. Kad, M. A. Meyers, Acta Mater. 78 (2014) 378-393.

[5] J. Kwon, M. C. Brandes, P. Sudharshan Phani, A. P. Pilchak, Y. F. Gao, E. P. George, G. M. Pharr, M. J. Mills, Acta Mater. 61 (2013) 4743-4756.

[6] R. Smith, D. Christopher, S. D. Kenny, A. Richter, B. Wolf, Phys. Rev. B 67 (2003) 245405.

[7] M. M. Biener, J. Biener, A. M. Hodge, A. V. Hamza, Phys. Rev. B 76 (2007) 165422.

[8] S. Das, H. Yu, E. Tarleton, F. Hofmann, Appl. Phys. Lett. 114 (2019) 221905.

[9] J. Alcalá, R. Dalmau, O. Franke, M. Biener, J. Biener, A. Hodge, Phys. Rev. Lett. 109 (2012) 075502.

[10] S. Roy, R. Gatti, B. Devincre, D. Mordehai, Comput. Mater. Sci. 162 (2019) 47-59.

[11] S. Lee, A. Vaid, J. Im, B. Kim, A. Prakash, J. Guénolé, D. Kiener, E. Bitzek, S. H. Oh, Nat. Commun. 11 (2020) 2367.

[12] I. A. Alhafez, C. J. Ruestes, Y. Gao, H. M. Urbassek, Nanotechnology 27 (2015) 045706.

[13] H. Xiang, H. Li, T. Fu, C. Huang, X. Peng, Acta Mater. 138 (2017) 131139.

[14] T. Fu, X. Peng, C. Wan, Z. Lin, X. Chen, N. Hu, Z. Wang, Appl. Surf. Sci. 392 (2017) 942-949.

[15] M. C. Fivel, C. F. Robertson, G. R. Canova, L. Boulanger, Acta Mater. 46 (1998) 6183-6194.

[16] H. Fan, A. H. W. Ngan, K. Gan, J. A. El-Awady, Int. J. Plast. 111 (2018) $152-167$.

[17] Y. Cui, G. Po, N. Ghoniem, Scr. Mater. 154 (2018) 34-39.

[18] L. B. Munday, J. C. Crone, J. Knap, Acta Mater. 103 (2016) 217-228.

[19] E. V. d. Giessen, A. Needleman, Model. Simul. Mat. Sci. Eng. 3 (1995) 689.

[20] H. Yu, A. Cocks, E. Tarleton, J. Mech. Phys. Solids. 123 (2019) 41-60.

[21] B. Bromage, E. Tarleton, Model. Simul. Mat. Sci. Eng. 26 (2018) 085007. 


\section{Supplementary data}

Supplementary data to this article can be found online. 